\title{
Oral and dental health in head and neck cancer survivors
}

\author{
Firoozeh Samim ${ }^{*}$, Joel B. Epstein ${ }^{2}$, Zachary S. Zumsteg ${ }^{3}$, Allen S. Ho ${ }^{4}$ and Andrei Barasch ${ }^{5}$
}

\begin{abstract}
Therapeutic improvements and epidemiologic changes in head and neck cancer (HNC) over the last three decades have led to increased numbers of survivors, resulting in greater need for continuing management of oral and dental health in this population. Generally, the HNC patient oral health needs are complex, requiring multidisciplinary collaboration among oncologists and dental professionals with special knowledge and training in the field of oral oncology. In this review, we focus on the impact of cancer treatment on oral health, and the oral care protocols recommended prior to, during and after cancer therapy. The management of oral complications such as mucositis, pain, infection, salivary function, taste and dental needs are briefly reviewed. Other complications and their management, including osteonecrosis of the jaw and recurrent/new primary malignancies are also described. This review offers clinical protocols and information for medical providers to assist in understanding oral complications and their management in HNC patients and survivors, and their oral and dental health care needs. Oral and dental care is impacted by the patient's initial oral and dental status, as well as the specific cancer location, type, and its treatment; thus, close communication between the dental professional and the oncology team is required for appropriate therapy.
\end{abstract}

Keywords: Cancer treatment protocol, Cancer pre-treatment protocol, Oral hygiene, Oral complication management, Head and neck cancer survivors

\section{Background}

More than 600,000 cases of HNC are diagnosed each year worldwide. With evolving etiologies and advances in treatment, HNC survival has improved in recent decades and the population of HNC survivors continues to grow [1]. This increasing number of survivors raises additional challenges, particularly in the management of those patients who have complex medical, oral/dental, and psychosocial needs. Management of the oral complications in this population typically requires multidisciplinary collaboration among different professionals and healthcare providers, including head and neck surgeons, medical oncologists, radiation oncologists, and dental professionals with special knowledge and training in the field of oral oncology.

HNC may present with oral manifestations, which necessitates recognition and appropriate referral/treatment

\footnotetext{
* Correspondence: f.samim@alumni.ubc.ca

'Department of Oral Medicine Oral Pathology, University of British Columbia, Vancouver, BC, Canada

Full list of author information is available at the end of the article
}

[2, 3]. Generally, therapy for malignant diseases affects the mouth both directly through cytotoxicity, and indirectly through the effects on immune function or other systemic side effects. Common oral complications include pain, mucositis, salivary gland dysfunction, taste loss/change, infections, dysphagia, fibrosis, soft tissue and/or bone necrosis, exacerbation of dental and periodontal diseases, and recurrent or secondary malignancy [4]. The incidence and severity of oral complications are affected by cancer treatment modality(s) used, anatomic location and stage of the cancer, extent of oral or dental diseases prior to treatment, comorbidities and genetic risk, oral hygiene and nutrition [5]. To effectively prevent as well as treat oral complications, dental professionals play important roles prior to, during and following active cancer treatment [6].

For HNC survivors, it is generally recognized that trained/experienced dental professionals serve as integral components of the multidisciplinary oncology team. These dental professionals can play a role in prevention of oral complications through patient education (improve oral 
hygiene, maintain nutrition, reduce alcohol and tobacco use), treatment of dental disease, prophylactic strategies and treatment of oral complications, and early detection of oral malignancy in high-risk patients [7]. The related referral pathways are through dental and medical specialists with oncology experience [8]. This review focuses on the role of dental health care providers, and protocols of oral care in $\mathrm{HNC}$ patients.

\section{Head and neck cancer treatment and its impact on the oral cavity}

All treatment modalities for HNC produce oral complications, including surgery (e.g. mutilation and physiologic changes), radiation therapy (e.g. mucositis, dysphagia, hyposalivation, osteoradionecrosis), and neoadjuvant, adjuvant and/or concurrent chemotherapy (e.g. mucositis, taste changes, immune suppression) [9]. Additionally, newer targeted therapies may also result in oral mucosal complications $[10,11]$. These medications include epidermal growth factor inhibitors, which cause erythematous mucosal reactions; tyrosine kinase inhibitors and mammalian target of rapamycin (mTOR) inhibitors, both of which may cause isolated aphthous-like lesions; and emerging immunotherapies, which may induce lichenoid reactions [12]. Given the commonality of oral complications of therapy, pretreatment oral conditions may affect therapeutic options selected for some patients [2]. Additionally, the long-term oral sequelae of these treatments require oral and dental follow-up and fastidious long-term oral care.

Oncologists need to recognize the importance of pretreatment dental care, and use available resources to effectively address oral needs [13]. For improved outcomes, it is imperative that a thorough oral assessment, implementation of basic oral care protocols, management of preexisting dental conditions, and prevention and management of emerging oral complications be completed before HNC treatment. This is best accomplished by integrated teams with oncology-trained/experienced dental providers who provide timely dental treatment and preventive protocols that do not disrupt cancer therapy.

The following sections provide a summary of evidencebased guidelines related to oral/dental management in HNC patients, with the goal of improving health outcomes and enhancing long-term quality of life.

\section{Before HNC therapy}

It is important to be aware that some practicing dentists may have limited experience in care of the oncology patient, and that dental professionals with oncology experience may be required to identify and manage oral conditions and diseases in HNC patients [14]. According to the Multinational Association of Supportive Care in Cancer/ International Society of Oral Oncology (MASCC/ISOO) guidelines, thorough basic oral care is recommended to all cancer patients for control of bacterial flora and reduction of inflammatory and/or infectious complications $[15,16]$. Oral hygiene recommendations are shown in Table 1.

HNC patients should receive a comprehensive oral assessment prior to any cancer treatment, as soon as possible after diagnosis, and ideally, within 2 to 3 weeks prior to beginning cytotoxic therapy, in order to allow time for healing if surgical dental procedures are indicated [17-19]. The assessment should include full periodontal examination, radiographic examination, salivary gland functional assessment, and jaw range of motion measurement. Other treatments, such as non-surgical dental needs and stabilizing dental conditions can be completed as needed, or postponed until after cancer therapy, if deemed elective [20, 21].

Individual $\mathrm{HNC}$ patients should be managed according to cancer therapy-specific pre-treatment dental protocols [22]. Absence of symptomatic and occult oral disease is the desired goal, and elimination of acute and chronic infections that may require future surgical care is indicated prior to cancer therapy. Oral tissues within the high dose radiation fields must receive particular attention, as postradiation surgery in this region may be risky. Custom oral devices (positioning/opening devices, midline blocks or anti-scatter trays) may be prescribed to minimize radiation exposure to oral structures unaffected by cancer and to assist in tissue positioning in order to support radiation exposure to movable tissues on a repeated basis. Compromises in timing and extent of dental treatment may be necessary, particularly when tumor burden is excessive, but generally, the healthier the mouth, the better the odds for good outcomes [21, 22].

\section{During HNC treatment}

It is essential that oncologists and other physicians involved in cancer care communicate with trained dental professionals in complex and unique cancer cases [23] so that necessary treatment is provided in timely fashion, unnecessary treatment is avoided, and preventive protocols are instituted. Even though cancer patients may be inclined to discontinue oral hygiene due to discomfort, the avoidance of basic hygiene results in increased microbial loads, gingival/oral inflammation and risk of infections. Thus, maintenance of oral hygiene should be encouraged. If oral hygiene is compromised during cancer treatment, the daily use of aqueous chlorhexidine $0.12 \%$ solution can control the overall microbial load, including fungal and yeast overgrowth [24]. Devices built for prophylactic purposes (midline blocks, custom trays, etc.), must be used according to protocol.

Oral pain, predominantly due to mucositis, is one of the major symptoms in radiation therapy with or without chemotherapy. The biology of mucositis has been described and is leading to study of interventions based on the 
Table 1 Oral care prior to and post cancer treatment in head and neck cancer survivors

\begin{tabular}{|c|c|}
\hline Pre - Cancer Treatment & $\begin{array}{l}\text { - Pre-treatment assessment 2-3 weeks prior to cancer therapy } \\
\text { o Comprehensive head and neck, oral mucosa, dental and periodontal examination } \\
\text { o Radiographs to assess dental and periodontal status } \\
\text { o Baseline jaw range of motion (interincisal opening), baseline resting and stimulated saliva } \\
\text { o Advanced caries, advanced periodontal disease: definitive treatment may require surgery } \\
\text { with goal of } 1-2 \text { weeks of healing time } \\
\text { o Periodontal debridement maintenance; oral hygiene instruction } \\
\text { o Custom fluoride carriers, custom oral positioning devices }\end{array}$ \\
\hline During Cancer Treatment & $\begin{array}{l}\text { o Individual treatment as cancer type and planned treatment indications } \\
\text { o Oral hygiene reinforced } \\
\text { o Small carious lesions may be treated with fluoride and/or sealants; daily fluoride applications } \\
\text { o Symptom management: Pain: topical analgesic and anesthetic agents; systemic analgesics; } \\
\text { dry mouth: hydration, oral rinses and coating agents; lip management } \\
\text { o Mucositis reduction: Patient education: } \\
\text { o Regular brushing, flossing; prosthesis cleaning } \\
\text { o Bland oral rinses, water based/wax or lanolin lip lubricant } \\
\text { o Fluoridated toothpaste; or home fluoride trays daily in high risk patients } \\
\text { o Soft toothbrushes; Electric or ultrasonic brushes for tolerated patients } \\
\text { o Super-soft brush for severe mucositis or foam brush with chlorhexidine if brushing not possible } \\
\text { o Dietary instruction; nutritional guidance, tobacco and alcohol avoidance }\end{array}$ \\
\hline Post - Cancer Treatment & $\begin{array}{l}\text { o Monitoring, prevention and management of oral complications (mucositis, dry mouth, } \\
\text { mucosal pain, taste change, infection, dental demineralization, dental caries, periodontal } \\
\text { disease, soft tissue/osteonecrosis etc.) } \\
\text { o Checking for cancer recurrence or secondary primary cancer } \\
\text { o Dental caries prevention, periodontal maintenance } \\
\text { o Determine frequency of dental hygiene follow-up interval based on level of hyposalivation, } \\
\text { demineralization/caries rate and patient's oral hygiene post-radiotherapy; patients with dry mouth, } \\
\text { may require hygiene and recall every } 3-4 \text { months } \\
\text { o Patient education } \\
\text { o Fluoridated toothpaste; in high risk patients home fluoride trays daily } \\
\text { o Good oral hygiene, soft toothbrushes or electric or ultrasonic brushes, flossing } \\
\text { o Maintain lubrication of mouth and lips } \\
\text { o Encourage non-cariogenic diet and cessation of tobacco \& alcohol }\end{array}$ \\
\hline
\end{tabular}

pathogenesis of the condition [25]. For pain management due to mucositis, MASCC/ISOO recommends topical analgesic/anesthetic agents (e.g., lidocaine, benzocaine, diphenhydramine), or in severe cases, patient-controlled analgesia (parenteral morphine sulphate). Study of potential prevention of mucositis is ongoing, with recent suggestion for use of low-energy laser (LLLT), which may significantly reduce the severity and duration of mucositis [2, 14]. Medications that affect neuropathic pain such as gabaminergic agents (e.g. gabapentin), or tricyclic antidepressants (e.g. imipramine, amitriptyline) should be considered in addition to systemic analgesics [26]. Pain management strategies for this group of patients are summarized in Table 2. In

Table 2 Pain management Techniques in oncology (modified from Epstein et al.; Orofacial pain in Cancer; Part II- Clinical Perspective and Management; J Dent Res 86(6)2007

Palliative Radiation Therapy

Topical Analgesics/topical anesthetics

Systemic analgesics

Adjunctive medications (eg: Anxiolytics, Anticonvulsants, Antidepressant)

Physical and rehabilitative therapy: (massage/Physiotherapy; Cold/moist heat compresses)

Photobiomodulation/acupuncture

Psychologist support (Cognitive behavioral therapy, hypnosis) addition, dietary instruction, frequent use of saline/bicarbonate rinses, and good oral hygiene should be addressed. Finally, a number of medical devices typically described as mucosal coating agents (eg: Biotene ${ }^{\oplus}$, Gelclair ${ }^{\circledR}$, Mugard ${ }^{\oplus}$, Gelclair ${ }^{\ominus}$ and others), has been cleared by the FDA but data supporting impact on mucositis are limited and no guidelines have been developed by MASSC/ISOO to date. Nevertheless, these over-the-counter coating agents may be soothing and promote temporary oral comfort.

In addition to mucositis, acute oral/dental infection during radiation therapy may be a cause for cancer treatment interruptions and excess morbidity. Extractions or other surgical procedures are not indicated during cytoreductive therapy. Hence, the alternative to surgical treatment is medical management, using antibiotics and analgesics that may control, but not resolve the infection, and create additional issues for the patient. Dental disease prevention must include regular daily fluoride applications, best applied via custom-made fluoride trays; when not able to be used (e.g. if severe mucositis develops), application by brushing on teeth or rinse application can be substituted. Fluoride application must be continued on a daily basis as long as dry mouth persists. In these patients, it is also important to provide a topical calcium source for the teeth in addition to fluoride. Special toothpastes (e.g. Enamelon ${ }^{\bullet}$ ) have been designed for this purpose. 
Patient education is an integral part of dental treatment in order to support nutrition, reduce alcohol and tobacco use, and receive optimal oral care, which are important for both oral health and effective completion of cancer therapy [27, 28]. In addition, other factors such as taste changes, mucosal sensitivity, and xerostomia may negatively impact diet and nutritional intake, which may affect oral and general health. As a consequence, oral symptoms need attention from both oncologists and dental professionals in order to ensure timely diagnosis and appropriate treatment, as well as patient compliance with treatment recommendations.

\section{Post HNC treatment}

Cytotoxic treatment-induced oral complications can be severe, and impact not only the quality of life, but also cancer therapy outcomes. This can be challenging, as patients are known to under-report oral complications to their medical providers during and following cancer therapy. HNC patients should be monitored closely to reinforce prevention, early diagnosis and management of late complications, and optimal oral care to prevent these complications. Timely identification of cancer recurrence and/or metastasis, leading to early referral is also indicated [29]. Effective management of oral infection, residual mucositis, sensory changes (mucosal pain, taste change/loss), reduced/altered salivation, dental and periodontal disease, soft tissue/bone necrosis, and temporomandibular joint disorders must be integral part of post-cancer therapy care [30]. Follow-up dental visits should be tailored and individualized based on patient conditions. At least twice per year check-ups are recommended, although an every 2-3 month schedule may be indicated for some cases (Table 1).

Patients should be instructed in daily atraumatic tooth brushing, bland oral rinses, flossing and fluoride gel applications, as well as management of mucositis and other residual sequelae, based on the MASCC/ISOO evidencebased guidelines [2, 11]. Ultrasonic or electric brushes may be recommended, although soft or supersoft manual toothbrushes are standard [31]. Prescription toothpaste/gel with $5000 \mathrm{ppm}$ fluoride are recommended for dentate patients, but if the patient cannot tolerate due to mucositis, fluoride rinses may be substituted for short periods; return to brushing with prescription toothpaste should be conducted as soon as possible and maintained for the duration of the patient's dentate life.

Patients skilled at dental flossing should be instructed to continue, avoiding trauma to the gingival tissue. Other interdental cleaning aids may be chosen if they are effective, and can be used atraumatically [32, 33]. Diet and supplements that are rich in carbohydrates, as well as sucrose-sweetened medications should be avoided, or when needed to support energy intake, should be taken with meals, and may be best after oral hygiene is performed [34].

\section{Management of specific oral complications related to $\mathrm{HNC}$ \\ Mucositis}

Mucositis is recognized as a critical, dose-limiting toxicity of current HNC cytotoxic therapy and its prevalence approaches $100 \%$. Acute signs and symptoms typically develop during second or third week of therapy and may continue for weeks to months following completion of treatment, particularly in those patients treated with chemoradiation [35]. Chronic mucosal sensitivity may be increased in those who experienced severe acute mucosal damage [33]. Radiation-induced loss of stem cells in the basal layer interferes with the replacement of cells in the superficial mucosal layers when they are lost through normal physiologic sloughing. The subsequent denuding of the epithelium results in mucositis, which can be painful and interfere with oral intake and nutrition. Chemotherapy can have a similar effect on the mucosa. Patient education includes frequent use of non-medicated bland oral rinses, possible use of film forming, lubricating mucosal coating agents, and topical analgesics/anesthetics as needed. Mouthwashes with alcohol, foods with acidic or spicy qualities, and coarse or abrasive foods should be avoided [36, 37]. Thorough and consistent oral hygiene is recommended.

Active research continues to evaluate novel prevention and treatment approaches to mucositis for future clinical application. Current management is focused on palliation of symptoms with topical and systemic analgesics. It is also important to recognize that pain due to mucositis is related to tissue damage and inflammation, resulting in nociceptive pain, but also due to neuropathic sensitization (neuropathic pain), which can be treated with neurologically active medications such as gabapentin and doxepin [37-41].

\section{Oral mucosal infections - viral, bacterial and fungal infections}

Patient monitoring is critical in diagnosis of viral, bacterial and fungal infections. Diagnosis can be challenging during the period of mucositis, as there may be considerable overlap in signs and symptoms of mucositis and infection. Viral infections such as Herpes simplex virus (HSV), and other herpes viruses are common in patients treated with chemotherapy, while HSV reactivation is uncommon in $\mathrm{HNC}$ patients treated with radiation alone. Acyclovir and valcyclovir are considered equally effective in HSV prevention and treatment $[42,43]$. Oral manifestations of viral infection may be more severe, with altered presentation and a more protracted course in cancer survivors. The more severe reactivation of 
Varicella-Zoster virus (shingles) requires larger doses of antiviral agents, which may be combined with systemic corticosteroids.

Bacterial infection occurs most commonly in association with dental or periodontal infection that may worsen during cancer treatment. For suitable selections of antibiotics (begin on an empiric basis for oral and dental infection), bacterial culture and antibacterial sensitivity tests may be required due to potential shifts in oral colonization, particularly in hospitalized patients, and in patients not responding to therapy as expected [34].

Oral yeast infection, most commonly due to Candida albicans is found in 7.5, 39.1 and $32.6 \%$ prior to, during and post HNC therapy, respectively [44]. Clinical presentation of oropharyngeal candidiasis may be variable and not easily recognized, presenting as white patches, erythematous patches or plaques, as hyperplastic candidiasis or mixed presentation [44]. Recognition of infection may be challenging due to multiple presentations and overlap with other mucosal changes such as mucositis. Other symptoms such as coated sensation in the mouth, burning sensation and taste change can be associated with candidiasis and should raise attention.

Topical therapy for local disease is always preferable, but systemic antifungals may be necessary in the setting of immune deficiency or loss of mucosal integrity. Oncologists need to be aware that topical antifungal agents have had inconsistent efficacy and some are high in sugar, which reduces efficacy and increases dental caries risk in dry mouth dentate patients. Antifungals that are not sugar sweetened (e.g. vaginal suppositories), and oral systemic agents are more effective [45, 46]. Furthermore, additional fungal species with increased resistance to antifungal agents are observed in cancer patients, and therefore the principles of culture and identification of the fungal species may be important to guide therapy, particularly in non-responding cases [44].

\section{Other oral complications and their management}

Other oral complications in HNC survivors are common. The key is to identify risk factors and early onset symptoms, and to offer referral for expert care. For example, HNC patients receiving cytotoxic chemotherapy and radiotherapy can have dry mouth or xerostomia that continues indefinitely due to reduced saliva production (hyposalivation) [47, 48]. Dry mouth is a common and significant chronic complication and drives late oral complications; some patients may accommodate to ongoing hyposalivation over time and may report reduced or even no symptoms. However, effects of reduced saliva on the health of oral tissues continue. Hyposalivation management includes assessment of saliva production, with first approach to treatment being stimulation of any residual function (chewing and taste stimulation, systemic sialagogues such as pilocarpine, civemiline, or bethanechol), and palliative topical products [49]. In addition to reduced production of saliva, changes in the quality of saliva, with thickened or viscous secretions can be challenging to manage, and trials of mucolytic agents may be considered (guaifenesin, acetyl-cysteine). Encouraging good daily oral hygiene, frequent dental visits, and dental fluoride and remineralization supplementation can help counteract the changes induced by insufficient salivation. For those patients with persisting hyposalivation, wetting of oral surfaces, and replacement of calcium, phosphate, and use of antimicrobial rinses have been shown to be safe and effective in relieving symptoms of dry mouth and minimizing infection potential. Dry mouth-associated taste change, gastrointestinal upset, mucosal sensitivity, inflammation and ulceration [50] significantly limit food intake, and may lead to dietary compromise that complicates nutrition and general health. Dry mouth increases risk of dental demineralization and tooth cavitation that can lead to rampant dental damage, increase risk for progression of periodontal disease and increase risk of osteoradionecrosis of the jaw (ORN). Dental sensitivity to temperature is also associated with lower saliva secretion and lower salivary $\mathrm{pH}$. Oral burning sensation due to mucosal sensitivity is associated with hyposalivation and arises from peripheral neuropathy [2, 51]. In addition, 50$75 \%$ of HNC patients have dysgeusia, which contributes to the reduction in quality of life. Radiation- and chemotherapy-induced neuropathies are associated with inflammation, neurotoxicity, oxidative stress, and ischemia, which may persist long after clinical mucositis resolved [52, 53].

Among other complications, ORN is a known risk following head and neck radiotherapy. It is associated with dental disease and dental procedures, but can occur spontaneously, without identified stimulus. This risk may be increased in people on medications such as bisphosphonates or denosumab for osteoporosis, people with diabetes, on immunosuppressives, and in tobacco users [54]. Prevention of ORN is the key concept and it reinforces the need for dental stabilization before cancer therapy, and continuing good oral/dental health in survivors. Management of ORN after onset must be individualized in coordinated dental and medical care.

Current treatment strategies are based on limited research and clinical anecdotes and thus, should be undertaken by experienced healthcare providers due to the complexity of care. Hyperbaric oxygen (HBO) therapy and use of antiseptic oral rinses, and sometimes antibiotics when secondary infection is diagnosed, has been the medical approach to therapy [55]. However, the use of HBO is expensive and controversial and no good evidence for or against it has been published. More recently medical management with local infection management and 
pentoxifylline and Vitamin $\mathrm{E}$ has been supported in phase II clinical trials [51]. If necrosis develops, continuation of these medications is recommended with the addition of clodronate to stimulate new bone formation [56]. As necrotic bone cannot be regained, the goal of treatment is prevention of progression, pain control, and management of secondary infection in hard and soft tissues (osteomyelitis, cellulitis). Surgical management with local sequestrectomy or vascularized tissue transfer may be needed [57].

Post treatment fibrosis is best managed by early recognition and physical therapy. Pentoxifylline and vitamin E has been suggested [58].

Recurrent or second primary oral malignancy is a known risk and people with prior upper aerodigestive tract cancer are the highest risk population for a post-treatment malignant lesion. Post-radiation mucosal changes can make detection of early changes difficult, and the potentially delayed healing in the high dose radiation fields make determination to biopsy more challenging.

Speech and swallow therapists and nutrition/dietary instruction are important aspects of care in the HNC patient, and these professionals are important part of the multidisciplinary HNC team.

\section{Conclusion}

The number of HNC survivors has increased, due mostly to change in risk factors for cancer (HPV), and advances in cancer therapeutics and supportive care. This growing population requires specialized oral care by personnel trained in managing the intricacies of these complex patients. Oncologists and dentists must collaborate to optimize care, as well as to increase knowledge of preventive and therapeutic options for oral health maintenance, oral cancer detection and suitable referral conditions with pathways. The oncology team should include dental professionals specialized in head and neck cancer survivor management, which will contribute to better oral complication prevention, detection, and treatment, and an improved quality of life.

\section{Abbreviations \\ HNC: Head and neck cancer; HPV: Human papilloma virus; HSV: Herpes simplex virus; LLLT: Low-energy laser; MASCC/ISOO: Multinational Association of Supportive Care in Cancer/International Society of Oral Oncology; mTOR: Mammalian target of rapamycin; ONJ: Osteoradionecrosis of the jaw}

\section{Funding}

Not applicable. There was no funding for this review paper.

\section{Authors' contribution}

FS and JE initiated the review paper, each author contributed to writing and review of the entire review paper. All authors edited, read and reviewed the paper prior to submission.

\section{Competing interests}

The authors declare that they have no competing interests.

\section{Data}

Not applicable. As this is a review paper there is no data to be shared.

\section{Author details}

'Department of Oral Medicine Oral Pathology, University of British Columbia, Vancouver, BC, Canada. ${ }^{2}$ Samuel Oschin Comprehensive Cancer Institute, Cedars-Sinai Medical Center, Los Angeles, CA, USA. ${ }^{3}$ Radiation Oncology, Samuel Oschin Comprehensive Cancer Institute, Cedars-Sinai Medical Center, Los Angeles, CA, USA. ${ }^{4}$ Department of Surgery, Samuel Oschin

Comprehensive Cancer Institute, Cedars-Sinai Medical Center, Los Angeles, CA, USA. ${ }^{5}$ Department of Medicine, Weill Cornell Medical College, New York, NY, USA.

Received: 18 May 2016 Accepted: 3 October 2016

Published online: 19 October 2016

\section{References}

1. Baxi SS, Pinheiro LC, Pati SM, Pfister DG, Oeffinger KC, Elkin EB. Causes of death in long term survivors of head and neck cancer. Cancer. 2014;120:1507-13.

2. Epstein JB, Thariat J, Bensadoun RJ, Barasch A, Murphy BA, Kolnick L, Popplewell L, Maghami E. Oral complications of cancer and cancer therapy: from cancer treatment to survivorship. CA Cancer J Clin. 2012;62(6):400-22.

3. Islam NM, Bhattacharyya I, Cohen DM. Common oral manifestations of systemic disease. Otolaryngol Clin North Am. 2011;44(1):161-82.

4. Epstein JB, Raber-Drulacher J, Wilkins A, et al. Advances in hematologic stem cell transplant: an update for oral health care providers. Oral Surg Oral Med Oral Pathol Oral Radiol Endod. 2009;107:301-12.

5. Bethesda MD. Oral Complications of Chemotherapy and Head/Neck Radiation. National Cancer Institute. 2013. https://www.cancer.gov/about-cancer/ treatment/side-effects/mouth-throat/oral-complications-hp-pdq\#section/_13. Accessed 5 Oct 2016

6. Sankaranarayanan R, Ramadas K, Thomas G, et al. Trivandrum Oral Cancer Screening Study Group. Effect of screening on oral cancer mortality in Kerala, India: A cluster-randomised controlled trial. Lancet. 2005;365(9475):1927-33.

7. American Dental Hygienists' Association. Access to care position paper. Chicago: American Dental Hygienists' Association; 2001. https://www.cdha.ca/pdfs/ Profession/Resources/position_paper_access_angst.pdf. Accessed 5 Oct 2016.

8. Epstein JB, Güneri P, Barasch A. Appropriate and necessary oral care for people with cancer: guidance to obtain the right oral and dental care at the right time. Support Care Cancer. 2014;22(7):1981-8.

9. Perterson DE, Jensen SB. Oral complications of nonsurgical cancer therapies: diagnosis and treatment. In: Glick M, editor. Burket's Oral Medicine. 12th ed. Shelton: PMPH-USA, Ltd; 2014. p. 201-18.

10. Thariat J, Vignot S, Lapierre A, et al. Integrating genomics in head and neck cancer treatment: Promises and pitfalls. Crit Rev Oncol Hematol. 2015;95(3):397-406.

11. Jackson LK, Johnson DB, Sosman JA, Murphy BA, Epstein JB. Oral health in oncology: impact of immunotherapy. Support Care Cancer. 2015;23(1):1-3.

12. Ackson LK, Johnson DB, Sosman JA, Murphy BA, Epstein JB. Oral health in oncology: impact of immunotherapy. Support Care Cancer. 2015;23:1-3. doi:10.1007/s00520-014-2434-6.

13. Ganzer $H$, Epstein JB, Touger-Decker R, et al. Nutrition management of the cancer patient. In: Touger-Decker R, Mobley C, Epstein JB, editors. Nutrition and Oral Medicine. New York: Human Press, Springer; 2014. p. 235-53. https://www.youtube.com/embed/MnignqBw4CY. Accessed 5 Oct 2016.

14. Oral Complications of Chemotherapy and Head/Neck Radiation-for health professionals $\left(\mathrm{PDQ}^{\oplus}\right)$ https:/www.cancer.gov/about-cancer/treatment/sideeffects/mouth-throat/oral-complications-pdq. Accessed 5 Oct 2016

15. Lalla RV, Bowen J, Barasch A, Elting L, Epstein J, et al. Mucositis Guidelines Leadership Group of the Multinational Association of Supportive Care in Cancer and International Society of Oral Oncology (MASCC/ISOO). MASCC/ ISOO clinical practice guidelines for the management of mucositis secondary to cancer therapy. Cancer. 2014;120(10):1453-61.

16. Elad S, Raber-Durlacher JE, Brennan MT. Basic oral care for hematologyoncology patients and hematopoietic stem cell transplantation recipients: a position paper from the joint task force of the Multinational Association of Supportive Care in Cancer/International Society of Oral Oncology (MASCC/ ISOO) and the European Society for Blood and Marrow Transplantation (EBMT). Support Care Cancer. 2015;23(1):223-36.

17. National Institute of dental and craniofacial research; Oral Complications of Cancer Treatment: What the Dental Team Can Do. http://www.nidcr.nih. gov/oralhealth/Topics/CancerTreatment/OralComplicationsCancerOral.htm. Accessed 5 Oct 2016. 
18. Epstein JB, Ransier A, Sherlock CH. Acyclovir prophylaxis of oral herpes virus during bone marrow transplantation. Eur J Cancer B Oral Oncol. 1996;32:158-62.

19. Jackson LK, Epstein JB, Migliorati CA, et al. Development of tools for the oral health and panoramic evaluation of the head and neck cancer patient: A methodological study. Spec Care Dentist. 2015. doi:10.1111/scd.12125. [Epub ahead of print].

20. Rankin K, Jones D, Redding S, et al. Oral Health in Cancer Therapy: A Guide for Health Care Professionals; third edition. 2008. http://www.exodontia.info/ files/Oral_Health_in_Cancer_Therapy_-_A_Guide_for_Health_Care_ Professionals_3rd_edition.pdf. Accessed 5 Oct 2016.

21. Schubert MM, Peterson DE. Oral complications of hema-topoietic cell transplantation. In: Appelbaum RF, Forman SJ, Negrin RS, Blume KG, editors. Thomas' Hematopoietic Cell Transplantation: Stem Cell Transplantation. 4th ed. Oxford: Wiley-Blackwell; 2009. p. 1589-607.

22. Lalla RV, Brennan MT, Schubert MM. Oral complications of cancer therapy. In: Yagiela JA, Dowd FJ, Johnson BS, Marrioti AJ, Neidle EA, editors. Pharmacology and Therapeutics for Dentistry. 6th ed. St. Louis: MosbyElsevier; 2011. p. 782-98.

23. Joshi VK. Dental treatment planning and management for the mouth cancer patient. Oral Oncol. 2010;46(6):475-9.

24. Elad S, Epstein JB, von Bultzingslowen I, Drucker S, Tzach R, Yarom N. Topical immunomodulators for management of oral mucosal conditions, a systematic review; Part II: miscellaneous agents. Expert Opin Emerg Drugs. 2011;16:183-202.

25. Villa A, Sonis ST. Mucositis: pathobiology and management. Curr Opin Oncol. 2015;27:159-64.

26. Epstein JB, Elod S, Eliav E, Jurevic R, Benoliel R. Orofacial pain in cancer: Part II-Clinical Perspective and Management. J Dent Res. 2007:86:6-506.

27. National Cancer Institute. Oral Complications of Chemotherapy and Head/ Neck Radiation. Bethesda: National Cancer Institute; 2013. http://www. webmd.com/cancer/tc/oral-complications-of-chemotherapy-and-headneckradiation-pdq-supportive-care-health-professional-information-nci-aboutthis-pdq-summary?page=2. Accessed 5 Oct 2016.

28. Ganzer H, Touger-Decker R, Parrott JS, Murphy BA, Epstein JB, Huhmann MB. Symptom burden in head and neck cancer: impact upon oral energy and protein intake. Support Care Cancer. 2013;21(2):495-503.

29. Kasperts N, Slotman B, Leemans CR, Langendijk JA. A review on re-irradiation for recurrent and second primary head and neck cancer. Oral Oncol. 2005; 41(3):225-43.

30. Bensinger W, Schubert M, Ang KK, et al. NCCN Task Force Report, Prevention and management of mucositis in cancer care. J Natl Compr Canc Netw. 2008;6(Suppl 1):S1-21.

31. Hong $\mathrm{CH}$, daFonseca M. Considerations in the pediatric population with cancer. Dent Clin N Am. 2008:52(1):155-81.

32. Ransier A, Epstein JB, Lunn R, Spinelli J. A combined analysis of a toothbrush, foam brush, and a chlorhexidine-soaked foam brush in maintaining oral hygiene. Canc Nurs. 1995;18(5):393-6.

33. Keefe DM, Schubert MM, Elting LS, et al. Updated clinical practice guidelines for the prevention and treatment of mucositis. Cancer. 2007;109(5):820-31.

34. Hong $\mathrm{CH}$, Napeñas JJ, Hodgson $\mathrm{BD}$, et al. A systematic review of dental disease in patients undergoing cancer therapy. Support Care Cancer. 2010;18(8):1007-21.

35. Elting LS, Cooksley CD, Chambers MS, Garden AS. Risk, outcomes and costs of Radiation induced oral mucositis among patients with head and neck malignancies. Int J Radiat Oncol Biol Phys. 2007;68(4):1110.

36. Lalla RV, Sonis ST, Peterson D. Management of oral mucositis in patient with cancer. Dent Clin North Am. 2008:52(1):61.

37. Sonis ST. Pathobiology of oral mucositis: novel insights and opportunities. J Support Oncol. 2007:5(9 Suppl 4):3-11.

38. Epstein JB, Saunders DP. Managing Oral Mucositis Cancer Therapy. 2015. http://www.oralhealthgroup.com/features/managing-oral-mucositis-cancertherapy/. Accessed 5 Oct 2016.

39. Epstein JB, Epstein JD, Epstein MS, Oien H, Truelove EL. Doxepin rinse for management of mucositis pain in patients with cancer: one week follow-up of topical therapy. Spec Care Dentist. 2008;28(2):73-7

40. Saunders DP, Epstein JB, Elad S, Allemano J, Bossi $P$, et al. Systematic review of antimicrobials, mucosal coating agents, anesthetics and analgesics for the management of oral mucositis in cancer patients. Support Care Cancer. 2013; 21:3191-207

41. Kataoka T, Kiyoita N, Shimada T, Funakoshi Y, Charahara N, et al. Randomized trial of standard pain control with or without gabapentin for pain related to radiation-induced mucositis in head and neck cancer. Auris Nasus larynx. 2016;43(6):677-84.
42. Reusser P. Management of viral infections in immunocompromised cancer patients. Swiss Med Wkly. 2002;132(27-28):374-8.

43. Arduino PG, Porter SR. Oral and perioral herpes sim- plex virus type 1 (HSV1) infection: review of its management. Oral Dis. 2006;12(3):254-70.

44. Lalla RV, Latortue MC, Hong CH, et al. A systematic review of oral fungal infections in patients receiving cancer therapy. Support Care Cancer. 2010; 18(8):985-92.

45. Worthington HV, Clarkson JE, Khalid T, et al. Interventions for treating oral candidiasis for patients with cancer receiving treatment. Cochrane Database Syst Rev. 2010;7:CD001972

46. Gøtzche PC, Johansen HK. Nystatin prophylaxis and treatment in severely immunocompromised patients. Cochrane Database Syst Rev. 2002;2:CD002033.

47. Nieuw Amerongen AV, Veerman EC. Current therapies for xerostomia and salivary gland hypofunction associated with cancer therapies. Support Care Cancer. 2003:11(4):226-31.

48. Epstein JB, Beier Jensen S. Management of Hyposalivation and Xerostomia: Criteria for Treatment Strategies. Compend Contin Educ Dent. 2015;36(8):600-3.

49. Turner MD, Ship JA. Dry mouth and its effects on the oral health of elderly people. J Am Dent Assoc. 2007;138(Suppl):15S-20.

50. Watters AL, Epstein JB, Agulnik M. Oral complications of targeted cancer therapies: a narrative literature review. Oral Oncol. 2011;47(6):441-8.

51. Saunders DP, Epstein JB, Elad SA, et al. For The Mucositis Study Group of the Multinational Association of Supportive Care in Cancer/International Society of Oral Oncology (MASCC/ISOO). Systematic review of antimicrobials, mucosal coating agents, anesthetics, and analgesics for the management of oral mucositis in cancer patients. Support Care Cancer. 2013;21(11):3191-207.

52. Dropcho EJ. Neurotoxicity of radiation therapy. Neurol Clin. 2010;28(1):217-34.

53. Cooperstein E, Gilbert J, Epstein JB, et al. Vanderbilt Head and Neck Symptom Survey version 2.0: report of the development and initial testing of a subscale for assessment of oral health. Head Neck. 2012;34:797-804

54. Ruggiero SL, Dodson TB, Fantasia J, et al. American Association of Oral and Maxillofacial Surgeons Position Paper on Medication-Related Osteonecrosis of the Jaw-2014 Update. J Oral Maxillofac Surg. 2014;72:1938-56.

55. Bennett MH, Feldmeier J, Hampson N, Smee R, Milross C. Hyperbaric oxygen therapy for late radiation tissue injury. Cochrane Database Syst Rev. 2012;(5): CD005005.

56. Robard L, Louis MY, Blanchard D, Babin E, Delanian S. Medical treatment of osteoradionecrosis of the mandible by PENTOCLO: preliminary results. Eur Ann Otorhinolaryngol Head Neck Dis. 2014;131:333-8.

57. Gal T, Futran NC. Influence of prior hyperbaric oxygen therapy in complications following microvascular reconstruction for advanced osteoradionecrosis. Arch Otol Head Neck Surg. 2003;129:72-6.

58. Zecha JA, Raber-Durlacher JE, Nair RG, Epstein JB, et al. Low-level laser therapy/ photobiomodulation in the management of side effects of chemoradiation therapy in head and neck cancer: part 1: proposed applications and treatment protocols. Support Care Cancer. 2016;24:2793-805

\section{Submit your next manuscript to BioMed Central and we will help you at every step:}

- We accept pre-submission inquiries

- Our selector tool helps you to find the most relevant journal

- We provide round the clock customer support

- Convenient online submission

- Thorough peer review

- Inclusion in PubMed and all major indexing services

- Maximum visibility for your research

Submit your manuscript at www.biomedcentral.com/submit 